\title{
Some Integrals Relating to the $I_{e}$-Function
}

\section{By Shigehiko Okui}

Abstract. Various integrals relating to the $I_{e}$-function

$$
I_{e}(k, z)=\int_{0}^{z} e^{-x} I_{0}(k x) d x,
$$

which finds a wide variety of applications in the fields of statistical communication theory and noise analysis, are evaluated in closed form.

1. Introduction. Rice [1] in his study of statistical properties of a sine wave influenced by Gaussian noise, presented a number of basic relations concerning the $I_{e}$-function. The above function yields the Nakagami-Hoyt probability distribution function [2], and hence it is often encountered in problems of statistical communication theory and noise analysis [3], [4], [5], [6]. In the present paper, we evaluate in closed form certain integrals involving the $I_{e}$-function, and also others reducible to it. Many of these results are believed to be new.

Short statements on derivations are presented in the Appendix. The notations for the special functions involved are consistent with those given in works by Erdelyi [7], [8] and Gradshteyn and Ryzhik [9]. Additional references [10], [11], [12], [13], [14] are also available for properties of integrals of Bessel functions.

2. Integrals Involving the $I_{e}$-Function.

$$
\begin{gathered}
\int_{0}^{z} e^{-p x} I_{e}(k, x) d x=\frac{1}{p}\left\{\frac{1}{p+1} I_{e}\left(\frac{k}{p+1},[p+1] z\right)-e^{-p z} I_{e}(k, z)\right\} \\
\int_{0}^{z} e^{-p x} I_{e}(k, x) x d x \\
=\frac{1}{p}\left[\left\{\frac{1}{p(p+1)}+\frac{1}{(p+1)^{2}-k^{2}}\right\}\right. \\
\quad \cdot I_{e}\left(\frac{k}{p+1},[p+1] z\right)-\left(z+\frac{1}{p}\right) e^{-p z} I_{e}(k, z) \\
\left.-\frac{z}{(p+1)^{2}-k^{2}} e^{-(p+1) z}\left\{k I_{1}(k z)+(p+1) I_{0}(k z)\right\}\right]
\end{gathered}
$$

$$
\int_{0}^{z} I_{e}(k, x) d x=\left(z-\frac{1}{1-k^{2}}\right) I_{e}(k, z)+\frac{z e^{-z}}{1-k^{2}}\left\{I_{0}(k z)+k I_{1}(k z)\right\} \text {. }
$$

Received October 27, 1982; revised February 28, 1983.

1980 Mathematics Subject Classification. Primary 33A40; Secondary 33A35, 94A05. 
(2.4)

$$
\begin{aligned}
\int_{0}^{z} I_{e}(k, x) x d x=\frac{1}{2}[ & \left\{z^{2}-\frac{2+k^{2}}{\left(1-k^{2}\right)^{2}}\right\} I_{e}(k, z)+\frac{z e^{-z}}{1-k^{2}} \\
& \left.\cdot\left\{\left(z+\frac{2+k^{2}}{1-k^{2}}\right) I_{0}(k z)+k\left(z+\frac{3}{1-k^{2}}\right) I_{1}(k z)\right\}\right]
\end{aligned}
$$

(2.5) $\int_{0}^{1} I_{e}(k, x)(1-x)^{\nu-1} d x$

$$
=\frac{1}{\nu(\nu+1)} \Phi_{2}\left[\frac{1}{2}, \frac{1}{2} ; \nu+2 ;-(1-k),-(1+k)\right], \quad \nu>0 .
$$

(2.6) $\int_{0}^{1} I_{e}(k, x[1-x]) x d x=\frac{1}{12} \Phi_{2}\left(\frac{1}{2}, \frac{1}{2} ; \frac{5}{2} ;-\frac{1-k}{4},-\frac{1+k}{4}\right)$.

$$
\begin{aligned}
\int_{0}^{\infty} e^{-p x} I_{e}(k, x) x^{\nu-2} d x= & \frac{\Gamma(\nu)}{p^{\nu}} F_{1}\left(\nu ; \frac{1}{2}, \frac{1}{2} ; 2 ;-\frac{1-k}{p},-\frac{1+k}{p}\right), \\
& p>k-1 \text { if } k \geqslant 1, p>0 \text { if } 1>k>0 ; \nu>0 .
\end{aligned}
$$

$$
\begin{aligned}
& \int_{0}^{\infty} e^{-p x} I_{e}(k, x) d x=\frac{1}{p \sqrt{(p+1)^{2}-k^{2}}}, \\
& p>k-1 \text { if } k \geqslant 1, p>0 \text { if } 1>k>0 .
\end{aligned}
$$

(2.9) $\int_{0}^{\infty} e^{-p x} I_{e}(k, x) x d x=\frac{(p+1)(2 p+1)-k^{2}}{p^{2} \sqrt{\left\{(p+1)^{2}-k^{2}\right\}^{3}}}$,

$$
p>k-1 \text { if } k \geqslant 1, p>0 \text { if } 1>k>0 .
$$

(2.10) $\int_{0}^{\infty} e^{-p x} I_{e}(k, x) x^{2} d x$

$$
\begin{array}{r}
=\frac{3 p+2-k^{2}}{p^{3} \sqrt{\left\{(p+1)^{2}-k^{2}\right\}^{3}}}-\frac{3(p+1)\left[(p+1)(2 p+1)-k^{2}\right]}{p^{2} \sqrt{\left\{(p+1)^{2}-k^{2}\right\}^{5}}}, \\
p>k-1 \text { if } k \geqslant 1, p>0 \text { if } 1>k>0 .
\end{array}
$$

(2.11) $\int_{0}^{\infty} e^{-p x} I_{e}(k, x) x^{-1} d x$

$$
\begin{aligned}
& =\frac{1}{\sqrt{k^{2}-1}}\left\{\sin ^{-1}\left(\frac{1}{k}\right)-\sin ^{-1}\left(\frac{p+1-k^{2}}{p k}\right)\right\}, \quad k>1, p>k-1 ; \\
& =\sqrt{\frac{p+2}{p}}-1, \quad k=1, p>0 ; \\
& =\frac{1}{\sqrt{1-k^{2}}} \ln \left[\frac{p+\sqrt{1-k^{2}}\left\{\sqrt{(p+1)^{2}-k^{2}}+\sqrt{1-k^{2}}\right\}}{p\left(1+\sqrt{1-k^{2}}\right)}\right], \\
& 1>k>0, p>0 .
\end{aligned}
$$


(2.12) $\int_{0}^{\infty} I_{e}(k, x) x^{-(\nu+1)} d x=\frac{\Gamma(1-\nu)}{\nu}{ }_{2} F_{1}\left(\frac{1-\nu}{2}, \frac{2-\nu}{2} ; 1 ; k^{2}\right)$,

$$
1>k>0,1>\nu>0 \text {. }
$$

(2.13) $\int_{0}^{\infty} I_{e}\left(k, x^{2}\right) x^{-2} d x=\kappa \sqrt{\frac{2}{\pi k}} K(\kappa), \quad$ where $\kappa^{2}=\frac{2 k}{1+k}, 1>k>0$.

(2.14) $\int_{0}^{\infty} I_{e}\left(k, x^{2}\right) J_{1}(2 a x) d x$

$$
=\frac{1}{2 a \sqrt{1-k^{2}}} \exp \left(-\frac{a^{2}}{1-k^{2}}\right) I_{0}\left(\frac{k a^{2}}{1-k^{2}}\right), \quad 1>k>0 \text {. }
$$

$$
\begin{array}{r}
\int_{0}^{\infty} I_{e}(k, x) J_{\nu+1}(a x) x^{-\nu} d x=\frac{a^{\nu-1}}{2^{\nu} \Gamma(\nu+1)} F_{4}\left(\frac{1}{2}, 1 ; \nu+1,1 ;-a^{2}, k^{2}\right), \\
1>k>0, \nu>-\frac{1}{2} .
\end{array}
$$

$$
\begin{aligned}
& \int_{0}^{\infty} I_{e}(k, x) J_{1}(a x) d x=\frac{2}{\pi} \sqrt{\frac{\kappa \kappa^{\prime}}{a^{3} k}} K(\kappa), \\
& \text { where } \kappa^{2}=\frac{1}{2}\left\{1-\frac{1-k^{2}+a^{2}}{\sqrt{\left(1-k^{2}+a^{2}\right)^{2}+4 a^{2} k^{2}}}\right\}, \\
& \kappa^{\prime 2}=1-k^{2}, 1>k>0 .
\end{aligned}
$$

$$
\begin{aligned}
\int_{0}^{\infty} I_{e}(k, x) K_{1}(k x) d x= & \frac{2}{k(1+2 k)} K(\kappa), \\
& \quad \text { where } \kappa^{2}=\left(\frac{1-2 k}{1+2 k}\right)^{2}, 1>k>0 .
\end{aligned}
$$

\section{Integrals Reducible to the $I_{t}$-Function.}

$$
\begin{gathered}
\int_{0}^{z} e^{-p x} I_{0}(a x) d x=\frac{1}{p} I_{c}(a / p, p z) . \\
\int_{0}^{z} e^{-p x} I_{0}(a x) x d x \\
=\frac{1}{p^{2}-a^{2}}\left[I_{e}(a / p, p z)-z e^{-p z}\left\{a I_{1}(a z)+p I_{0}(a z)\right\}\right] .
\end{gathered}
$$

(3.3) $\int_{0}^{z} e^{-p x} I_{0}(a x) x^{2} d x$

$$
\begin{aligned}
& =\frac{1}{p\left(p^{2}-a^{2}\right)^{2}}\left[\left(2 p^{2}+a^{2}\right) I_{e}(a / p, p z)\right. \\
& -p z e^{-p z}\left\{p\left(p^{2}-a^{2}\right) z+2 p^{2}+a^{2}\right\} I_{0}(a z) \\
& \left.-a p z e^{-p z}\left\{\left(p^{2}-a^{2}\right) z+3 p\right\} I_{1}(a z)\right] .
\end{aligned}
$$

$$
\int_{0}^{z} e^{-p x} I_{1}(a x) d x=\frac{1}{a}\left[I_{e}(a / p, p z)+e^{-p z} I_{0}(a z)-1\right] \text {. }
$$


(3.5)

$$
\begin{aligned}
& \int_{0}^{z} e^{-p x} I_{1}(a x) x d x \\
& \quad=\frac{1}{p\left(p^{2}-a^{2}\right)}\left[a I_{e}(a / p, p z)-p z e^{-p z}\left\{p I_{1}(a z)+a I_{0}(a z)\right\}\right]
\end{aligned}
$$

(3.6)

$$
\begin{aligned}
\int_{0}^{z} e^{-p z} I_{2}(a x) x d x & \\
=\frac{1}{a^{2}\left(p^{2}-a^{2}\right)}[ & \left(3 a^{2}-2 p^{2}\right) I_{e}(a / p, p z) \\
& -a^{3} z e^{-p z} I_{1}(a z)-\left\{a^{2} p z+2\left(p^{2}-a^{2}\right)\right\} e^{-p z} I_{0}(a z) \\
& \left.+2\left(p^{2}-a^{2}\right)\right]
\end{aligned}
$$

$$
\begin{aligned}
\int_{0}^{z} e^{-p x^{2}} I_{0}(a x) x d x= & \frac{1}{4 p} \exp \left(\frac{a^{2}}{4 p}\right)\left\{1-\frac{a^{2}-4 p^{2} z^{2}}{a^{2}+4 p^{2} z^{2}} I_{e}(k, Z)\right\} \\
& -\frac{1}{4 p} \exp \left(-p z^{2}\right) I_{0}(a z),
\end{aligned}
$$

where in (3.7)-(3.13),

$$
k=\frac{4 a p z}{a^{2}+4 p^{2} z^{2}}, \quad Z=p z^{2}+\frac{a^{2}}{4 p}, \quad p>0 .
$$

(3.8) $\int_{0}^{z} e^{-p x^{2}} I_{0}(a x) x^{3} d x$

$$
\begin{aligned}
= & \frac{1}{4 p^{2}}\left(1+\frac{a^{2}}{4 p}\right) \exp \left(\frac{a^{2}}{4 p}\right)\left\{1-\frac{a^{2}-4 p^{2} z^{2}}{a^{2}+4 p^{2} z^{2}} I_{e}(k, Z)\right\} \\
& -\frac{1}{4 p^{2}} \exp \left(-p z^{2}\right)\left\{\left(1+\frac{a^{2}}{4 p}+2 p z^{2}\right) I_{0}(a z)+a z I_{1}(a z)\right\} .
\end{aligned}
$$

$$
\begin{aligned}
\int_{0}^{z} e^{-p x^{2}} I_{1}(a x) d x= & \frac{1}{2 a} \exp \left(\frac{a^{2}}{4 p}\right)\left\{1-\frac{a^{2}-4 p^{2} z^{2}}{a^{2}+4 p^{2} z^{2}} I_{e}(k, Z)\right\} \\
& +\frac{1}{2 a} \exp \left(-p z^{2}\right) I_{0}(a z)-\frac{1}{a} .
\end{aligned}
$$

$$
\begin{aligned}
& \text { (3.10) } \begin{aligned}
\int_{0}^{z} e^{-p x^{2}} I_{1}(a x) x^{2} d x & =\frac{a}{8 p^{2}} \exp \left(\frac{a^{2}}{4 p}\right)\left\{1-\frac{a^{2}-4 p^{2} z^{2}}{a^{2}+4 p^{2} z^{2}} I_{e}(k, Z)\right\} \\
& -\frac{a}{8 p^{2}} \exp \left(-p z^{2}\right)\left\{I_{0}(a z)+4 \frac{p z}{a} I_{1}(a z)\right\} . \\
\text { (3.11) } \int_{0}^{z} e^{-p x^{2}} I_{2}(a x) x d x= & \left(\frac{1}{4 p}-\frac{1}{a^{2}}\right) \exp \left(\frac{a^{2}}{4 p}\right)\left\{1-\frac{a^{2}-4 p^{2} z^{2}}{a^{2}+4 p^{2} z^{2}} I_{e}(k, Z)\right\} \\
& -\left(\frac{1}{4 p}+\frac{1}{a^{2}}\right) \exp \left(-p z^{2}\right) I_{0}(a z)+\frac{2}{a^{2}} .
\end{aligned}
\end{aligned}
$$


(3.12) $\int_{0}^{z} e^{-p x^{2}} I_{2}(a x) x^{3} d x$

$$
\begin{aligned}
= & \frac{a^{2}}{16 p^{3}} \exp \left(\frac{a^{2}}{4 p}\right)\left\{1-\frac{a^{2}-4 p^{2} z^{2}}{a^{2}+4 p^{2} z^{2}} I_{e}(k, Z)\right\} \\
& -\frac{a^{2}}{16 p^{3}} \exp \left(-p z^{2}\right)\left\{\left(1+8 \frac{p^{2} z^{2}}{a^{2}}\right) I_{0}(a z)+4 \frac{p z}{a}\left(1-4 \frac{p}{a^{2}}\right) I_{1}(a z)\right\} .
\end{aligned}
$$

(3.13) $\int_{0}^{z} e^{-p x^{2}} I_{3}(a x) x^{2} d x$

$$
\begin{aligned}
= & \frac{a}{8 p^{2}}\left(1-8 \frac{p}{a^{2}}+32 \frac{p^{2}}{a^{4}}\right) \exp \left(\frac{a^{2}}{4 p}\right)\left\{1-\frac{a^{2}-4 p^{2} z^{2}}{a^{2}+4 p^{2} z^{2}} I_{e}(k, Z)\right\} \\
& -\frac{a}{8 p^{2}} \exp \left(-p z^{2}\right)\left\{\left(1-8 \frac{p}{a^{2}}-32 \frac{p^{2}}{a^{4}}\right) I_{0}(a z)+4 \frac{p z}{a} I_{1}(a z)\right\}-\frac{8}{a^{3}} .
\end{aligned}
$$

(3.14) $\int_{0}^{\infty} J_{0}(a x) \ln \left(1+\frac{b^{2}}{x^{2}}\right) \cos (c x) d x=\frac{\pi}{c} I_{e}\left(\frac{a}{c}, b c\right), \quad c>a>0, b>0$.

(3.15) $\int_{0}^{\infty} J_{1}(a x) \ln \left(1+\frac{b^{2}}{x^{2}}\right) \sin (c x) d x$

$$
=\frac{\pi}{a}\left\{I_{e}\left(\frac{a}{c}, b c\right)+e^{-b c} I_{0}(a b)-1\right\}, \quad c>a>0, b>0 .
$$

(3.16) $\int_{0}^{\infty} J_{1}(a x) \tan ^{-1}(b / x) \cos (c x) d x$

$$
=\frac{\pi}{2 a}\left\{1-e^{-b c} I_{0}(a b)-I_{e}\left(\frac{a}{c}, b c\right)\right\}, \quad c>a>0, b>0 .
$$

(3.17) $\int_{0}^{\infty} J_{0}(a x) \tan ^{-1}(b / x) \sin (c x) d x=\frac{\pi}{2 c} I_{e}\left(\frac{a}{c}, b c\right), \quad c>a>0, b>0$.

$$
\int_{0}^{\infty} e^{-p x^{2}} J_{1}(a x) J_{0}(b x) d x=\frac{1}{2 a}\left\{1-e^{-Z} I_{0}\left(\frac{a b}{2 p}\right)+\frac{a^{2}-b^{2}}{a^{2}+b^{2}} I_{e}(k, Z)\right\}
$$

where in (3.18)-(3.21),

$$
\begin{array}{r}
k=\frac{2 a b}{a^{2}+b^{2}}, \quad Z=\frac{a^{2}+b^{2}}{4 p}, \quad p>0 . \\
\int_{0}^{\infty} e^{-p x^{2} J_{1}(a x) J_{1}(b x) x^{-1} d x} \\
=\frac{1}{4}\left\{\frac{a}{b}+\frac{b}{a}+\left(\frac{b}{a}-\frac{a}{b}\right) \frac{a^{2}-b^{2}}{a^{2}+b^{2}} I_{e}(k, Z)\right\} \\
\quad-\frac{1}{2} e^{-Z}\left\{\frac{1}{2}\left(\frac{a}{b}+\frac{b}{a}\right) I_{0}\left(\frac{a b}{2 p}\right)+I_{1}\left(\frac{a b}{2 p}\right)\right\} . \\
=\frac{1}{a^{2}}\left\{1-\left(1+\frac{a^{2}}{2 p}\right) e^{-Z} I_{0}\left(\frac{a b}{2 p}\right)+\frac{a^{2}-b^{2}}{a^{2}+b^{2}} I_{e}(k, Z)\right\} .
\end{array}
$$

(3.20) $\int_{0}^{\infty} e^{-p x^{2}} J_{2}(a x) J_{0}(b x) x d x$ 
618

SHIGEHIKO OKUI

(3.21)

$$
\begin{aligned}
& \int_{0}^{\infty} e^{-p x^{2}} J_{2}(a x) J_{1}(b x) d x \\
& =\frac{b}{2 a^{2}}\left[1-e^{-Z}\left\{I_{0}\left(\frac{a b}{2 p}\right)+2 \frac{a}{b} I_{1}\left(\frac{a b}{2 p}\right)\right\}+\frac{a^{2}-b^{2}}{a^{2}+b^{2}} I_{e}(k, Z)\right] . \\
& \int_{0}^{\infty} e^{-p x^{2}} I_{0}(a x) \operatorname{erf}(b x) x d x \\
& =\frac{b}{2 p}\left[\frac{2 \sqrt{p+b^{2}}}{p+2 b^{2}} \exp \left(\frac{a^{2}}{4 p}\right) I_{e}(k, Z)\right. \\
& \left.+\frac{1}{\sqrt{p+b^{2}}} \exp \left\{\frac{a^{2}}{8\left(p+b^{2}\right)}\right\} I_{0}\left[\frac{a^{2}}{8\left(p+b^{2}\right)}\right]\right]
\end{aligned}
$$

where in (3.22)-(3.24),

$$
k=\frac{p}{p+2 b^{2}}, \quad Z=\frac{a^{2}}{8 p}\left(1+\frac{b^{2}}{p+b^{2}}\right), \quad p>0 .
$$

$$
\int_{0}^{\infty} e^{-p x^{2}} I_{1}(a x) \operatorname{erf}(b x) d x=\frac{2 b \sqrt{p+b^{2}}}{a\left(p+2 b^{2}\right)} \exp \left(\frac{a^{2}}{4 p}\right) I_{e}(k, Z) .
$$

$$
\begin{aligned}
& \int_{0}^{\infty} e^{-p x^{2}} I_{2}(a x) \operatorname{erf}(b x) x d x \\
& =\frac{b}{p}\left[\frac{\sqrt{p+b^{2}}}{p+2 b^{2}}\left(1-4 \frac{p}{a^{2}}\right) \exp \left(\frac{a^{2}}{4 p}\right) I_{e}(k, Z)\right. \\
& \left.+\frac{1}{2 \sqrt{p+b^{2}}} \exp \left\{\frac{a^{2}}{8\left(p+b^{2}\right)}\right\} I_{0}\left[\frac{a^{2}}{8\left(p+b^{2}\right)}\right]\right] .
\end{aligned}
$$

$$
\int_{0}^{\infty} e^{-p x^{2}} I_{0}\left(a x^{2}\right) J_{1}(b x) d x=\frac{\sqrt{p^{2}-a^{2}}}{p b} I_{e}(k, Z),
$$

where in (3.25)-(3.31),

$$
k=\frac{a}{p}, \quad Z=\frac{p b^{2}}{4\left(p^{2}-a^{2}\right)}, \quad p>a>0 .
$$

(3.26)

$$
\begin{aligned}
& \int_{0}^{\infty} e^{-p x^{2}} I_{0}\left(a x^{2}\right) J_{2}(b x) x d x \\
= & \frac{2 \sqrt{p^{2}-a^{2}}}{p b^{2}} I_{e}(k, Z)-\frac{1}{2 \sqrt{p^{2}-a^{2}}} \exp \left\{-\frac{p b^{2}}{4\left(p^{2}-a^{2}\right)}\right\} I_{0}\left[\frac{a b^{2}}{4\left(p^{2}-a^{2}\right)}\right] .
\end{aligned}
$$


(3.27) $\int_{0}^{\infty} e^{-p x^{2}} I_{1}\left(a x^{2}\right) J_{0}(b x) x d x$

$$
\begin{aligned}
=\frac{1}{2 a}\left[\frac{p}{\sqrt{p^{2}-a^{2}}} \exp \left\{-\frac{p b^{2}}{4\left(p^{2}-a^{2}\right)}\right\}\right. & I_{0}\left[\frac{a b^{2}}{4\left(p^{2}-a^{2}\right)}\right] \\
& \left.+\frac{\sqrt{p^{2}-a^{2}}}{p} I_{e}(k, Z)-1\right] .
\end{aligned}
$$

(3.28) $\int_{0}^{\infty} e^{-p x^{2}} I_{1}\left(a x^{2}\right) J_{1}(b x) d x$

$$
\begin{aligned}
=\frac{b}{4 a}\left[\frac{1}{\sqrt{p^{2}-a^{2}}} \exp \left\{-\frac{p b^{2}}{4\left(p^{2}-a^{2}\right)}\right\}\right. & \\
& \cdot\left\{p I_{0}\left[\frac{a b^{2}}{4\left(p^{2}-a^{2}\right)}\right]+a I_{1}\left[\frac{a b^{2}}{4\left(p^{2}-a^{2}\right)}\right]\right\} \\
& \left.+\frac{\sqrt{p^{2}-a^{2}}}{p} I_{e}(k, Z)-1\right] .
\end{aligned}
$$

(3.29) $\int_{0}^{\infty} e^{-p x^{2}} I_{1}\left(a x^{2}\right) J_{2}(b x) x^{-1} d x$

$$
\begin{aligned}
=\frac{b^{2}}{16 a}\left[\frac{1}{\sqrt{p^{2}-a^{2}}} \exp \left\{-\frac{p b^{2}}{4\left(p^{2}-a^{2}\right)}\right\}\right. & \\
& \cdot\left\{\left(p-4 \frac{a^{2}}{b^{2}}\right) I_{0}\left[\frac{a b^{2}}{4\left(p^{2}-a^{2}\right)}\right]+a\left(1-4 \frac{p}{b^{2}}\right) I_{1}\left[\frac{a b^{2}}{4\left(p^{2}-a^{2}\right)}\right]\right\} \\
& \left.+\frac{\sqrt{p^{2}-a^{2}}}{p}\left(1+16 \frac{a^{2}}{b^{4}}\right) I_{e}(k, Z)-1\right] .
\end{aligned}
$$

(3.30) $\int_{0}^{\infty} e^{-p x^{2}} I_{1}\left(a x^{2}\right) J_{3}(b x) d x$

$$
\begin{aligned}
=4 \frac{a}{b^{3}}\left[\frac{\sqrt{p^{2}-a^{2}}}{p} I_{e}(k, Z)\right. & -\frac{b^{2}}{4 a \sqrt{p^{2}-a^{2}}} \exp \left\{-\frac{p b^{2}}{4\left(p^{2}-a^{2}\right)}\right\} \\
& \left.\cdot\left\{p I_{1}\left[\frac{a b^{2}}{4\left(p^{2}-a^{2}\right)}\right]+a I_{0}\left[\frac{a b^{2}}{4\left(p^{2}-a^{2}\right)}\right]\right\}\right] .
\end{aligned}
$$




$$
\begin{aligned}
\int_{0}^{\infty} e^{-p x^{2}} I_{2}\left(a x^{2}\right) J_{0}(b x) x^{3} d x & \frac{1}{8 a^{2} \sqrt{\left(p^{2}-a^{2}\right)^{5}}} \exp \left\{-\frac{p b^{2}}{4\left(p^{2}-a^{2}\right)}\right\} \\
& \cdot\left[\left\{4 a^{2} p\left(p^{2}-a^{2}\right)-a^{2} b^{2}\left(p^{2}+a^{2}\right)-8 p\left(p^{2}-a^{2}\right)^{2}\right\}\right. \\
& \left.\left.+\frac{a I_{0}}{4\left(p^{2}-a^{2}\right)}\right]+2 a^{3} b^{2} p I_{1}\left[\frac{a b^{2}}{4\left(p^{2}-a^{2}\right)}\right]\right] \\
+ & \frac{1}{a^{2}}\left\{1-\frac{\sqrt{p^{2}-a^{2}}}{p} I_{e}(k, Z)\right\} .
\end{aligned}
$$

Appendix-Derivations. In this appendix derivations of the above results are summarized:

(2.1) Integrate by parts, with $u=I_{e}(k, x)$ and $d v=e^{-p x} d x$.

(2.2) Differentiate (2.1) with respect to $p$.

(2.3), (2.4) Integrate by parts. Use (3.2) and (3.3), respectively.

(2.5), (2.6) Give series expansions for $I_{e}(k, x)$ in powers of $x$. The results follow by use of the Beta integral. Refer to [7, art. 5.7.1 (21)]; [8, appendices]; or [9, art. 9.261 (2)] for definition of $\Phi_{2}$.

(2.7) Use the series expansions in powers of $x$. Refer to [7, art. 5.7.1 (6)]; [8, appendices]; or [9, art. 9.180 (1)] for definition of $F_{1}$.

(2.8) This is a limiting case of $(2.1)$ as $z \rightarrow \infty$.

(2.9)-(2.11) Differentiate and integrate (2.8), respectively, with respect to $p$.

(2.12) Integrate by parts, with $u=I_{e}(k, x)$ and $d v=x^{-(\nu+1)} d x$.

(2.13) Let $\nu=\frac{1}{2}$ in (2.12). Refer to [15, art. 1.13 (13.5)].

(2.14) Integrate by parts, with $u=I_{e}\left(k, x^{2}\right)$ and $d v=J_{1}(a x) d x$. Employ [16, (71)].

(2.15) Integrate by parts, with $u=I_{e}(k, x)$ and $d v=J_{\nu+1}(a x) x^{-\nu} d x$. Employ [8, art. 4.16 (13)] after replacing $\alpha$ by $i \alpha$. Refer to [7, art. 5.7.1 (9)]; [8, appendices]; or [9, art. 9.180 (4)] for definition of $F_{4}$.

(2.16) This integral is a special case of (2.15) with $\nu=0$, in which [18, art. 4.1 (1)] is useful.

(2.17) Integrate by parts, with $u=I_{e}(k, x)$ and $d v=K_{1}(k x) d x$. Employ [17, art. $4.3(1)]$.

(3.1) Substitute $x=p t$ into the integral representation of the $I_{e}$-function in the Abstract and reidentify $k$ as $a / p$.

(3.2), (3.3) Differentiate (3.1) with respect to $p$.

(3.4) Integrate by parts, with $u=e^{-p x}$ and $d v=I_{1}(a x) d x$.

(3.5) Differentiate (3.4) with respect to $p$.

(3.6) Combine (3.2) and (3.4) via [9, art. 8.486 (1)].

(3.7) Use the relation between the Marcum's $Q$-function and the $I_{e}$-function. Refer to $[16,(25)]$. 
(3.8) Differentiate (3.7) with respect to $p$.

(3.9) Integrate by parts, with $u=e^{-p x^{2}}$ and $d v=I_{1}(a x) d x$.

(3.10) Integrate by parts, with $u=x I_{1}(a x)$ and $d v=x e^{-p x^{2}} d x$.

(3.11) Combine (3.7) and (3.9) via [9, art. 8.486 (1)].

(3.12) Combine (3.8) and (3.10) via [9, art. $8.486(1)]$.

(3.13) Combine (3.10) and (3.11) via [9, art. 8.486 (1)].

(3.14) Multiply $[9$, art. 6.695 (2)] by $\beta$, and then integrate with respect to $\beta$.

(3.15) Employ [9, art. 6.718 (3)] with $\nu=1$. Multiply both sides by $\beta$, and then integrate with respect to $\beta$.

(3.16) Employ [9, art. $6.718(4)]$ with $\nu=1$. Multiply both sides by $\beta^{2}$, and then integrate with respect to $\beta$.

(3.17) Integrate $[9$, art. 6.695 (3)] with respect to $\beta$.

(3.18) Combine [16, (21) and (25)].

(3.19) Combine (3.18) and (3.21) via [9, art. $8.486(1)]$.

(3.20) Employ [9, art. 6.633 (2)] with $p=0$, and then combine this result and (3.18) via [9, art. $8.486(1)]$.

(3.21) Use a procedure similar to that for (3.20). Here let $p=2$ and then combine it with (3.20).

(3.22) Integrate by parts, with $u=\operatorname{erf}(b x)$ and $d v=e^{-p x^{2}} I_{0}(a x) x d x$. Employ $[16,(10),(62)$ and $(25)]$.

(3.23) Integrate by parts, with $u=\operatorname{erf}(b x)$ and $d v=e^{-p x^{2}} I_{1}(a x) d x$. Employ [16, (14), (63) and (25)].

(3.24) Combine (3.22) and (3.23) via [9, art. $8.486(1)]$.

(3.25) Multiply [16, (71)] by $b$, and then integrate with respect to $b$.

(3.26) Combine (3.25) and [16, (71)] via [9, art. 8.473 (1)].

(3.27) Combine $[16,(72)$ and (25)].

(3.28) Combine [16, (80) and (25)].

(3.29) Combine (3.28) and (3.30) via [7, art. 7.2.8 (56)].

(3.30) Employ [9, art. 6.651 (6)] after letting $\nu=2$ and replacing $\beta$ by $i \beta$. Multiply this equation by $\gamma^{3}$, and then integrate it with respect to $\gamma$.

(3.31) Differentiate $[16,(71)]$ with respect to $q$. Combine this result and (3.27) via [9, art. $8.486(1)]$.

Acknowledgements. The author is indebted to Professor Toshihiko Namekawa and Assistant Professor Norihiko Morinaga of Osaka University for drawing his attention to this work. Thanks are also due to the referee for many helpful suggestions to improve the contents.

Department of lilectrical lingincering

Suzuka College of Tichnologn

Shiroko-cho, Suzuka-shi, Mic-kin

510-02 Japan

1. S. O. RicE, "Statistical propertics of a sine wave plus random noise," Bell System Tech. J., v. 27 , 1948, pp. 109-157.

2. M. NAKAgami, "On the intensity distribution $2 R / \sqrt{\alpha \beta} \exp \left[-R^{2} / 2(1 / \alpha+1 / \beta)\right]$ $\cdot I_{0}\left(R^{2} / 2[1 / \beta-1 / \alpha]\right)$ and its application to signal statistics," J. Res. Nat. Bur. Standards Sect. D, v. 68D, 1964, pp. $995-1003$.

3. W. R. Bennett \& J. R. Davey, Data Transmission, McGraw-Hill, New York, 1965. 
4. S. OKui, N. Morinaga \& T. Namekawa, "Statistical properties of maximal ratio combining diversity in correlated $m$-fading environments," Trans. IECE Japan, v. 62-B, 1979. pp. 283-290.

5. F. ADACHI, "Selection and scanning diversity effects in a digital FM land mobile radio with limiter discriminator detection," Trans. IECE Japan, v. 64-E, 1981, pp. 398-405.

6. R. F. Pawula, S. O. Rice \& J. H. Roberts, "Distribution of the phase angle between two vectors perturbed by Gaussian noise," IEEE Trans. Comm., v. COM-30, 1982, pp. 1828-1841.

7. A. ERdelyi Et Al. (ed.), Higher Transcendental Functions, Vols. 1-3, McGraw-Hill, New York, 1955.

8. A. ERdelyi et Al. (ed.), Tables of Integral Transforms, Vols. 1 and 2, McGraw-Hill, New York, 1955.

9. I. S. Gradshteyn \& I. M. Ryzhik, Tables of Integrals, Series, and Products, Corrected and enlarged edition, Academic Press, New York, 1980.

10. Y. L. LuKE, Integrals of Bessel Functions, McGraw-Hill, New York, 1962.

11. F. A. J. Ford, "Some infinite integrals involving products of Bessel functions," J. London Math. Soc., v. 41, 1966, pp. 728-730.

12. W. C. LindSEY, "Infinite integrals containing Bessel function products," J. Soc. Indust. Appl. Math., v. 12, 1964, pp. 458-464.

13. M. M. AGREST \& M. S. MAKSIMOv, Theory of Incomplete Cylindrical Functions and Their Applications, English edition, Springer-Verlag, Berlin and New York, 1971.

14. K. R. LASSEY, "On the computation of certain integrals containing the modified Bessel function $I_{0}(\xi)$," Math. Comp., v. 39, 1982, pp. 625-637.

15. F. Oberhettinger \& L. Badil, Tables of Laplace Transforms, Springer-Verlag, Berlin and New York, 1973.

16. A. H. Nutrall, Some Integrals Involving the Q-Function, NUSC Tech. Report 4297, 1972.

17. S. OKUI, "Complete elliptic integrals resulting from infinite integrals of Bessel functions," J. Res. Nat. Bur. Standards Sect. B., v. 78B, 1974, pp. 113-135.

18. S. OKUI, "Complete elliptic integrals resulting from infinite integrals of Bessel functions. II," J. Res. Nat. Bur. Standards Sect. B., v. 79B, 1975, pp. 137-170. 